\title{
Improvement of awareness, treatment and control of hypertension among chronic kidney disease patients in China from 1999 to 2005
}

\author{
Yu Wang ${ }^{1,2}$, Luxia Zhang ${ }^{1,2}$, Xiaomei $\mathrm{Li}^{1,2}$, Yulan $\mathrm{Xu}^{3}$, Min Yang ${ }^{4}$, Jiaqi Qian ${ }^{5}$, Lining Wang ${ }^{6}$, Nan $\mathrm{Chen}^{7}$, \\ Yong $\mathrm{Gu}^{8}$, Mangmang Chen ${ }^{9}$, Changying Xing ${ }^{10}$, Xiangmei Chen ${ }^{11}$, Fanfan Hou ${ }^{12}$, Xueqing $\mathrm{Yu}^{13}$, \\ Xiaomiao Cheng ${ }^{14}$, Lanzhong Guo ${ }^{15}$, Chongyi Wei ${ }^{16}$, Guodong Huang ${ }^{17}$, Qing Zhang ${ }^{18}$, Rong Wang ${ }^{19}$, \\ $\mathrm{Li}$ Wang ${ }^{20}$, Changlin $\mathrm{Mei}^{21}$, Youyun $\mathrm{Li}^{22}$, Zhihong $\mathrm{Liu}^{23}$, Liancheng Zhao ${ }^{24}$, Yangfeng $\mathrm{Wu}^{25}$ \\ and Hai Yan Wang ${ }^{1,2}$
}

Controlling hypertension is important to protect renal function and prevent cardiovascular disease in chronic kidney disease (CKD) patients. However, data on hypertension awareness, treatment and control among CKD patients are limited. Two nationwide surveys were conducted in China in 1999-2000 and 2004-2005 among, respectively, 1328 and 1244 adult, non-dialysis, hypertensive CKD patients, to assess the status of hypertension awareness, treatment and control and associated factors. A standard questionnaire was adopted, and blood pressure (BP) was measured by trained staff according to a standard protocol in both surveys. Compared with the data from 1999-2000, the data from 2004-2005 showed increased awareness ( 87.2 vs. $75.7 \%, P<0.001$ ), treatment ( 85.9 vs. $80.4 \%, P=0.001)$ and control $(30.0$ vs. $21.1 \%, P<0.001$, by the general threshold of $\mathrm{BP}<140 / 90 \mathrm{~mm} \mathrm{Hg} ; 7.7 \mathrm{vs.} 5.9 \%, P=0.075$, by an optimal threshold of $\mathrm{BP}<130 / 80 \mathrm{~mm} \mathrm{Hg}$ ) of hypertension. The odds ratios for general BP control were 1.4 (95\% confidence index (Cl), 1.1-1.7) for female gender, $1.1(95 \% \mathrm{Cl}, 1.0-1.1)$ for high estimated glomerular filtration rate, $1.3(95 \% \mathrm{Cl}, 1.1-1.6)$ for treatment in a local hospital, $2.8(95 \% \mathrm{Cl}, 2.0-3.9)$ for hypertension awareness and $1.7(95 \% \mathrm{Cl}, 1.4-1.9)$ for combined treatment. General physicians from local hospitals made greater contributions to the total improvement. Lack of treatment was mainly due to patients not recognizing the necessity for it. This is the first report of hypertension awareness, treatment and control among hypertensive CKD patients from a developing country. Improvement of awareness and general control of hypertension were demonstrated. Education of both physicians and patients regarding optimal BP control should be reinforced in the future.

Hypertension Research (2009) 32, 444-449; doi:10.1038/hr.2009.38

Keywords: awareness; chronic kidney disease; control; treatment

\section{INTRODUCTION}

Hypertension is an independent risk factor for the development of renal insufficiency and a major risk factor for cardiovascular disease
(CVD) morbidity and mortality related to chronic kidney disease (CKD).${ }^{1-4}$ It has been reported that the prevalence of hypertension in those with incipient renal insufficiency may be $30-60 \%$ and may rise

\footnotetext{
${ }^{1}$ Renal Division, Department of Medicine, Peking University First Hospital, Institute of Nephrology, Peking University, Beijing, China; ${ }^{2}$ Key Laboratory of Renal Disease, Ministry of Health of China, Beijing, China; ${ }^{3}$ Department of Nephrology, The First Affiliated Hospital of Wen Zhou Medical College, Wenzhou, China; ${ }^{4}$ Department of Nephrology, Li Hui Li Hospital of Ning Bo Medical Center, Ningbo, China; ${ }^{5}$ Department of Nephrology, Ren Ji Hospital, Shanghai Jiao Tong University School of Medicine, Shanghai, China; ${ }^{6}$ Department of Nephrology, The First Hospital of China Medical University, Shenyang, China; ${ }^{7}$ Department of Nephrology, Rui Jin Hospital, Shanghai Jiao Tong University School of Medicine, Shanghai, China; ${ }^{8}$ Department of Nephrology, Hua Shan Hospital, Fu Dan University, Shanghai, China; ${ }^{9}$ An Gang Tie Dong Hospital, Anshan, China; ${ }^{10}$ Department of Nephrology, The First Affiliated Hospital of Nanjing Medical University, Nanjing, China; ${ }^{11}$ Department of Nephrology, China PLA General Hospital, Beijing, China; ${ }^{12}$ Renal Division, Nanfang Hospital, Southern Medical University, Guangzhou, China; ${ }^{13}$ Department of Nephrology, The First Affiliated Hospital of Zhong Shan University, Guangzhou, China; ${ }^{14}$ Department of Nephrology, Xiangya Hospital, Central South University, Changsha, China; ${ }^{15}$ Dong Yang Chinese Taditional Medicine Hospital, Dongyang, China; ${ }^{16}$ The First Affiliated Hospital of Xin Xiang Medical School, Xingxiang, China; ${ }^{17}$ The Second Affiliated Hospital of Guang Xi Chinese Traditional Medicine College, Liuzhou, China; ${ }^{18}$ Department of Nephrology, Yan Tai Yu Huang Ding Hospital, Qing Dao University School of Medicine, Yantai, China; ${ }^{19}$ Department of Nephrology, Shandong Provincial Hospital, Jinan, China; ${ }^{20}$ Division of Nphrology, Si Chuan Provincial Hospital, Chengdu, China; ${ }^{21}$ Department of Nephrology, Shang Hai Chang Zheng Hospital, Shanghai, China; ${ }^{22}$ Affiliated Hospital of Luzhou Medical College, Luzhou, China; ${ }^{23}$ Department of Nephrology, Nanjing General Hospital of Nan Jing Millitary Command, Nanjing, China; ${ }^{24}$ Department of Epidemiology, Fu Wai Hospital and Cardiovascular Institute, Chinese Academy of Medical Science, Beijing, China and ${ }^{25}$ Department of Epidemiology and Biostatistics, School of Public Health, Peking University, Beijing, China

Correspondence: Dr X Li, Renal Division, Department of Medicine, Peking University First Hospital, Institute of Nephrology, Peking University, No. 8, Xishiku Street, Beijing 100034, China.

E-mail: xiaomei0708@gmail.com
}

Received 2 November 2008; revised 21 February 2009; accepted 27 February 2009 
to more than $80 \%$ in end-stage renal disease. ${ }^{5}$ Effective anti-hypertension therapy is critical to delay the decline of renal function ${ }^{6-11}$ and is associated with a significant reduction in CVD events in CKD patients. ${ }^{12-15}$ A report from the Joint National Committee on the Prevention, Detection, Evaluation, and Treatment of High Blood Pressure and the National Kidney Foundation Kidney Disease Outcome and Quality Initiative identified CKD patients as a high-risk group in whom intensive hypertension treatment is warranted. The report recommended that the blood pressure $(\mathrm{BP})$ of CKD patients be controlled to less than $130 / 80$, or $125 / 75 \mathrm{~mm} \mathrm{Hg}$ if proteinuria is higher than $1 \mathrm{~g}$ per day. ${ }^{16,17}$

Although hypertension control is very important in CKD patients, data on hypertension awareness, treatment and control among these patients are limited, ${ }^{18-20}$ whereas many studies have been performed in the general population. Furthermore, there are no studies from developing countries, where the medical care systems and medical resources are quite different from those in developed countries. The aim of the present study was to investigate hypertension awareness, treatment and control rates among non-dialysis hypertensive CKD patients in a developing country, China, and to analyze the possibility for improvement by comparing two surveys conducted in 1999-2000 and 2004-2005.

\section{METHODS}

\section{Study population}

The surveys were conducted in hospitals selected on the basis of geographical location and the socioeconomic status of the cities where they were located. The participating hospitals were divided into two types: type I hospitals had independent renal units in which CKD patients were followed by nephrologists, and type II hospitals were local hospitals where general physicians with special training in nephrology took care of CKD patients. The first survey, in 19992000, was performed in 20 hospitals, comprising 13 type I hospitals and 7 type II hospitals; a total of 1328 patients were recruited. The second survey, in 20042005, was conducted in 18 hospitals selected from those that took part in the first survey: of the 13 type I hospitals, two hospitals withdrew; of the 7 type II hospitals, 2 hospitals withdrew and 2 new hospitals joined in. A total of 1244 patients were recruited for the second survey. The reason for the withdrawal of hospitals in the second survey was that their chiefs were not interested in taking part in the survey again.

\section{Participant evaluation}

Adult hypertensive CKD patients from the selected hospitals aged 18 years or older and not on renal replacement treatment were invited to participate in the surveys. The surveys did not include patients who refused to participate or those with any of the following conditions: primary aldosteronism, pheochromocytoma, renal artery stenosis (vascular ultrasound or computed tomography renal arteriography was performed when clinical features such as a sudden increase in BP, refractory hypertension and elevation of serum creatinine ( $\mathrm{Scr}$ ) with a renin-angiotensin system inhibitor indicated the diagnosis), Cushing's syndrome, adrenal medullary hyperplasia, renin-secreting tumor, hyperthyroidism and pregnancy. All participants signed an informed consent form before they were asked by the medical staff to complete the standardized questionnaire. The study was approved by the Institutional Review Board of Peking University First Hospital. The questionnaire, which was administered and completed by trained medical staff, contained questions about basic demographic information (for example, gender, age, nationality), education, medical history of CKD (for example, clinical diagnosis of CKD cause, pathological diagnosis if renal biopsy was performed, regimen of immunosuppression treatment) and information about hypertension. Two BP readings were obtained with a mercury sphygmomanometer according to a standard protocol after the participant had been sitting quietly for $5 \mathrm{~min}$, with a 30 -s interval between every two measurements. The first and fifth Korotkoff sounds were recorded as systolic and diastolic BP, respectively. The average of the two readings was used for analysis. Measurements of Scr were made independently in each hospital and recorded in the questionnaire.

\section{Definitions}

CKD was diagnosed as a glomerular filtration rate of less than $60 \mathrm{ml} \mathrm{min}^{-1}$ per $1.73 \mathrm{~m}^{2}$ or the presence of kidney damage for more than 3 months, according to the Kidney Disease Outcomes Quality Initiative guidelines. ${ }^{21}$ The estimated glomerular filtration rate (eGFR) was calculated from Scr using the modified Modification of Diet in Renal Disease equation for Chinese. ${ }^{22}$ Patients were stratified into three groups according to eGFR: $\geqslant 60,30-60$ and $<30 \mathrm{ml} \mathrm{min}^{-1}$ per $1.73 \mathrm{~m}^{2}$.

Hypertension was defined as systolic BP $\geqslant 140 \mathrm{~mm} \mathrm{Hg}$ and/or diastolic BP $\geqslant 90 \mathrm{~mm} \mathrm{Hg}$, or undergoing current treatment with antihypertensive drugs. Awareness of hypertension was defined as the participant's knowledge of a previous diagnosis of hypertension before being invited to join the survey. Some patients were already taking anti-hypertensive drugs but were deemed unaware because they just followed the doctor's prescription without asking or being told why they should take the drugs. Treatment of hypertension was defined as use of antihypertensive drugs within the 2 weeks preceding the survey. Control was defined using two BP thresholds: less than $140 / 90 \mathrm{~mm} \mathrm{Hg}$ for the general population and less than $130 / 80 \mathrm{~mm} \mathrm{Hg}$ for CKD patients. Treatment with at least two types of antihypertensive drugs was defined as combined therapy.

Hospital type was defined as mentioned above. Education was divided into two subgroups: below or above high-school graduate. Chronic glomerulonephritis (CGN) was primarily diagnosed according to clinical features, including proteinuria, hematuria, edema and hypertension, when no evidence of any systemic disease such as systemic lupus erythematosus, which might cause these clinical features, could be found. Diabetic nephropathy was also primarily diagnosed clinically. Diabetic patients who had proteinuria as their prominent clinical presentation without obvious hematuria, usually accompanied by other clinical evidence of diabetic target organ injury such as retinopathy, were diagnosed as having diabetic nephropathy. Renal biopsy was not mandatory.

\section{Data collection}

The questionnaire used in both surveys was the same. It was designed by the Department of Epidemiology, Fu Wai Hospital and Cardiovascular Institute, Chinese Academy of Medical Science, the top institute in China for epidemiology studies, and the Renal Division, Department of Internal Medicine, Peking University First Hospital, the top institute in China for renal disease. Detailed descriptions of how to fill out the questionnaire were also attached to each questionnaire. Furthermore, there was a coordinator in every hospital who was strictly trained before administering the surveys by Peking University First Hospital regarding how to fill in the questionnaire according to the standardized protocol. These coordinators were responsible for training and supervising the local physicians participating in the study. Telephone communication was always open between the participating hospital and Peking University First Hospital if any problems were encountered during the surveys. The same method was used to collect data in both surveys, including the questionnaire and physical examination. All data were recorded on uniform record forms, coded and entered twice into computers by trained staff members at the Department of Epidemiology, Cardiovascular Institute, Chinese Academy of Medical Sciences for statistical analysis.

\section{Statistical analysis}

Continuous variables are presented as mean and standard deviation (s.d.), and categorical variables are presented as percentages. A $\chi^{2}$ test was used for categorical variables, while a $t$-test was used for continuous variables. Multiple logistic regression analysis was performed to assess the association of factors with awareness, treatment and control of hypertension. Variables for the awareness model included age, gender, education level, eGFR and hospital type. In addition, awareness of hypertension was added to the treatment model, and combination therapy was added to the control model. The survey year was also put into the above models as a variable. The results are reported as odds ratios (ORs) with a 95\% confidence index. Two-tailed $P$-values $<0.05$ were considered significant. 
All analyses were done using the SPSS statistical package, version 13.0 (SPSS, Inc., Chicago, IL, USA).

\section{RESULTS}

Demographic and clinical characteristics

A total of 2572 patients were enrolled in the two surveys, and about $23 \%$ of them had received a renal biopsy. The general characteristics of the participants are summarized in Table 1. In both surveys the most prevalent clinical diagnosis for CKD was primary CGN, which accounted for $54.3 \%$ of CKD patients in the first study and $54.9 \%$ in the second.

\section{Overall hypertension awareness, treatment and control rates}

From 1999 to 2005, both absolute hypertension awareness and treatment rates increased significantly (Table 2). Basing hypertension control on a less than $140 / 90 \mathrm{~mm} \mathrm{Hg}$ threshold, significant improvement was seen in the second survey, not only for those who were treated but also for all hypertensive participants. However, when the less than 130/80 $\mathrm{mm} \mathrm{Hg}$ threshold was used, the control rate was still poor and showed no significant improvement in the second survey.

There were strong unadjusted positive associations of the survey year 2004-2005 with hypertension awareness, treatment and control, using the year 1999-2000 as a reference. On adjustment for all clinically possible influencing factors (see details in Table 3 ), the OR of the 2004-2005 survey for hypertension awareness and control decreased but was still significant.

The stratified analysis of hypertension awareness, treatment and control by gender, age, education, eGFR and hospital type is shown in Table 4. In the 1999-2000 survey, women had better BP control at less than $140 / 90 \mathrm{~mm} \mathrm{Hg}$ than men, which was similar to the findings in an essential hypertension survey. However, in the 2004-2005 survey, both men and women showed significant improvement in BP control, and no difference could be found between them. Young participants $(<45$ years) had the least awareness of their hypertension in both surveys. $\mathrm{BP}$ control rates decreased in accordance with the decrease in eGFR levels in both surveys.

\section{Predictors of hypertension awareness, treatment and control} Multiple logistic regression analysis showed that older age (1.016 [1.009-1.023], $P<0.001)$ and higher education level (1.269 [1.0091.596], $P=0.041)$ were associated with better hypertension awareness. Hypertension awareness (10.355 [8.123-13.201], $P<0.001)$ was strongly associated with hypertension treatment. Female gender (1.356 [1.123-1.638], $P=0.001)$, high eGFR level (1.006 [1.0031.008], $P<0.001)$, type II hospital (1.336 [1.105-1.617], $P=0.003)$, hypertension awareness $(2.819[2.023-3.927), P<0.001)$ and combined antihypertensive drug therapy (1.667 [1.443-1.925], $P<0.001)$ were all independent predictors of BP less than $140 / 90 \mathrm{~mm} \mathrm{Hg}$.

\section{Contribution of general physicians at local hospitals to overall hypertension control}

Type II hospitals showed a significant increase in control rate for the threshold of less than $140 / 90 \mathrm{~mm} \mathrm{Hg}$ in 2004-2005 compared with 1999-2000 (37.0 vs. $21.1 \%, P<0.001$ ), whereas type I hospitals had similar control rates in both surveys ( 24.5 vs. $21.1 \%, P=0.13)$. Further analysis revealed that there was no gender difference between the patients from type II hospitals in each survey (female 47.1 vs. $48.5 \%$, $P=0.629$ ), but the participants in the 2004-2005 survey had higher eGFR levels than those in 1999-2000 (53.5 \pm 38.8 vs. $42.8 \pm 42.9 \mathrm{ml} \mathrm{min}^{-1}$ per $\left.1.73 \mathrm{~m}^{2}, P<0.001\right)$. Furthermore, there was significant improvement in hypertension awareness $(89.4$ vs. $73.9 \%$,
Table 1 Demographic and clinical characteristics of total participants

\begin{tabular}{|c|c|c|c|}
\hline Characteristics & $\begin{array}{c}1999-2000 \\
(N=1328)\end{array}$ & $\begin{array}{c}2004-2005 \\
(N=1244)\end{array}$ & ${ }^{*}$ P-value \\
\hline Women $(N, \%)$ & $619(46.6)$ & $578(46.5)$ & 0.94 \\
\hline Age (mean, s.d.) & $46.9(15.1)$ & $49.9(15.5)$ & $<0.001$ \\
\hline Age $(N, \%)$ & & & $<0.001$ \\
\hline$<45$ years & $612(46.1)$ & $486(39.1)$ & \\
\hline $45-60$ years & $392(29.5)$ & $386(31.0)$ & \\
\hline$>60$ years & $324(24.4)$ & $372(29.9)$ & \\
\hline Education (N, \%) & & & $<0.001$ \\
\hline$<$ High school & $782(59.4)$ & $320(26.0)$ & \\
\hline High-school graduate and above & $534(40.6)$ & $909(74.0)$ & \\
\hline eGFR (ml min ${ }^{-1}$ per $1.73 \mathrm{~m}^{2}$, mean (s.d.)) & $42.4(39.1)$ & $48.8(38.8)$ & $<0.001$ \\
\hline eGFR $(N, \%)$ & & & $<0.001$ \\
\hline$\geqslant 60 \mathrm{ml} \mathrm{min}^{-1}$ per $1.73 \mathrm{~m}^{2}$ & $394(29.7)$ & $426(34.2)$ & \\
\hline $30-60 \mathrm{ml} \mathrm{min}^{-1}$ per $1.73 \mathrm{~m}^{2}$ & $271(20.4)$ & $308(24.8)$ & \\
\hline$<30 \mathrm{ml} \mathrm{min}^{-1}$ per $1.73 \mathrm{~m}^{2}$ & $663(49.9)$ & $510(41.0)$ & \\
\hline Anti-hypertensive therapy (N, \%) & & & 0.01 \\
\hline Not taking & $213(16.0)$ & $202(16.2)$ & \\
\hline Single drug use & $457(34.4)$ & $361(29.0)$ & \\
\hline Combined drug use & $658(49.5)$ & $681(54.7)$ & \\
\hline Participants from hospital type II $(N, \%)$ & $645(48.6)$ & $546(43.9)$ & 0.017 \\
\hline
\end{tabular}

Abbreviations: eGFR, estimated glomerular filtration rate; s.d., standard deviation. ${ }^{*} P$-value for comparison between the years 1999-2000 and 2004-2005.

Table 2 Awareness, treatment and control of hypertension among hypertensive CKD patients

\begin{tabular}{cccr}
\hline & $\begin{array}{c}1999-2000 \\
(\mathrm{n}=1328)\end{array}$ & $\begin{array}{c}2004-2005 \\
(\mathrm{n}=1244)\end{array}$ & P-value \\
\hline Awareness $(N, \%)$ & $1005(75.7 \%)$ & $1085(87.2 \%)$ & $<0.001$ \\
Treatment $(N, \%)$ & $1068(80.4 \%)$ & $1065(85.9 \%)$ & 0.001 \\
Control $(N, \%)$ & & $373(30.0 \%)$ & $<0.001$ \\
Among all surveyed & & $96(7.7 \%)$ & 0.075 \\
$<140 / 90 \mathrm{~mm} \mathrm{Hg}$ & $280(21.1 \%)$ & & \\
$<130 / 80 \mathrm{~mm} \mathrm{Hg}$ & $79(5.9 \%)$ & $373(34.8 \%)$ & $<0.001$ \\
Among those treated & & $96(9.0 \%)$ & 0.174 \\
$<140 / 90 \mathrm{~mm} \mathrm{Hg}$ & $280(25.4 \%)$ & & \\
$<130 / 80 \mathrm{~mm} \mathrm{Hg}$ & $79(7.4 \%)$ & & \\
\hline
\end{tabular}

Abbreviation: CKD, chronic kidney disease.

$P<0.001$ ) and the rate of combined therapy ( 57.3 vs. $49.5 \%, P=0.017$ ) at local hospitals in the 2004-2005 survey.

After adjusting for eGFR, awareness of hypertension and combined therapy, the OR of the 2004-2005 survey for hypertension control in local hospitals was still significant, using the 1999-2000 survey as a reference (1.825 [1.392-2.394], $P<0.001)$.

\section{Causes for untreated patients}

In both surveys approximately $20 \%$ of patients did not receive any anti-hypertensive drugs. The most common reason for lack of treatment was that patients did not think it was necessary; this reason accounted for 54.0 and $71.3 \%$ of the untreated patients in the 19992000 and 2004-2005 surveys, respectively. Less common reasons included the absence of clear advice from physicians regarding sustained treatment, and forgetting to take medications. Financial limitations in covering the treatment expense accounted for only 3.5 and $3.1 \%$ of the untreated patients in the first and second survey, respectively. 
Table 3 Unadjusted and adjusted OR and 95\% Cl of awareness, treatment and control of hypertension in the year 2004-2005, with year 1999-2000 as the reference

\begin{tabular}{|c|c|c|c|}
\hline Adjusted factors & $\begin{array}{l}\text { Awareness } \\
(O R, 95 \% \mathrm{Cl})\end{array}$ & $\begin{array}{l}\text { Treatment } \\
(O R, 95 \% \mathrm{Cl})\end{array}$ & $\begin{array}{l}\text { Control } \\
(O R, 95 \% \mathrm{Cl})\end{array}$ \\
\hline Unadjusted & $2.152(1.747-2.652)$ & $1.430(1.158-1.766)$ & $1.603(1.340-1.917)$ \\
\hline Adjusted for age, gender & $2.073(1.680-2.557)$ & $1.392(1.126-1.721)$ & $1.616(1.349-1.936)$ \\
\hline Adjusted for age, gender, education & $1.899(1.513-2.384)$ & $1.206(0.958-1.519)$ & $1.651(1.357-2.009)$ \\
\hline Adjusted for age, gender, education, eGFR & $1.908(1.519-2.396)$ & $1.192(0.946-1.503)$ & $1.600(1.313-1.948)$ \\
\hline Adjusted for age, gender, education, eGFR, hospital type & $1.909(1.520-2.398)$ & $1.198(0.951-1.510)$ & $1.590(1.305-1.937)$ \\
\hline $\begin{array}{l}\text { Adjusted for age, gender, education level, eGFR, hospital type, } \\
\text { hypertension awareness }\end{array}$ & - & $0.885(0.684-1.146)$ & $1.461(1.196-1.786)$ \\
\hline $\begin{array}{l}\text { Adjusted for age, gender, education level, eGFR, hospital type, } \\
\text { hypertension awareness, combined therapy }\end{array}$ & - & - & $1.459(1.192-1.787)$ \\
\hline
\end{tabular}

Abbreviations: $\mathrm{Cl}$, confidence index; eGFR, estimated glomerular filtration rate; OR, odds ratio.

Survey 1999-2000 as reference; control for blood pressure $<140 / 90 \mathrm{~mm} \mathrm{Hg}$.

Table 4 Awareness, treatment and control of hypertension by gender, age, education level, eGFR and hospital type, 1999-2005

\begin{tabular}{|c|c|c|c|c|c|c|c|c|}
\hline & & & & & \multicolumn{4}{|c|}{ Control (\%) } \\
\hline & \multicolumn{2}{|c|}{ Awareness (\%) } & \multicolumn{2}{|c|}{ Treatment (\%) } & \multicolumn{2}{|c|}{$<140 / 90 \mathrm{~mm} \mathrm{Hg}$} & \multicolumn{2}{|c|}{$<130 / 80 \mathrm{~mm} \mathrm{Hg}$} \\
\hline & 1999-2000 & 2004-2005 & 1999-2000 & 2004-2005 & 1999-2000 & 2004-2005 & 1999-2000 & 2004-2005 \\
\hline \multicolumn{9}{|l|}{ Gender } \\
\hline Men & 75.6 & $86.6^{\ddagger}$ & 81.1 & 86.3 & 17.3 & $28.5^{\ddagger}$ & 4.9 & 7.4 \\
\hline Women & 76.7 & $87.8^{\ddagger}$ & 80.8 & 85.4 & $25.4^{\circ}$ & $31.7^{*}$ & 7.1 & 8.1 \\
\hline \multicolumn{9}{|l|}{ Age group } \\
\hline$<45$ years & 72.5 & $83.1^{\ddagger}$ & 77.7 & 84.5 & 21.4 & $30.2^{\dagger}$ & 6.7 & 7.8 \\
\hline $45-60$ years & $80.6^{\S}$ & $89.1^{\ddagger \#}$ & 83.8 & 86.8 & 21.7 & $32.6^{\ddagger}$ & 4.6 & $7.5^{*}$ \\
\hline$\geqslant 60$ years & $77.1^{\S}$ & $90.6^{\ddagger \#}$ & 83.6 & 86.8 & 19.8 & 26.9 & 6.2 & 7.8 \\
\hline \multicolumn{9}{|l|}{ Education } \\
\hline$<$ High school & 75.0 & $87.2^{\ddagger}$ & 78.6 & 85.6 & 22.6 & $30.9^{\dagger}$ & 6.3 & 9.1 \\
\hline High-school graduate and above & 77.9 & $87.3^{\ddagger}$ & 84.7 & 85.9 & 19.1 & 29.3 & 5.6 & 7.2 \\
\hline \multicolumn{9}{|l|}{ eGFR } \\
\hline$\geqslant 60 \mathrm{ml} / \mathrm{min} / 1.73 \mathrm{~m}^{2}$ & 71.5 & $86.6^{\ddagger}$ & 84.1 & $84.0^{\dagger}$ & 27.9 & 34.0 & 8.4 & 10.1 \\
\hline $30-60 \mathrm{ml} / \mathrm{min} / 1.73 \mathrm{~m}^{2}$ & 81.1 & $89.3^{\ddagger}$ & $80.0^{\#}$ & 89.3 & $18.8^{\bullet}$ & $32.1^{\dagger}$ & $5.5^{\#}$ & 7.5 \\
\hline$<30 \mathrm{ml} / \mathrm{min} / 1.73 \mathrm{~m}^{2}$ & 76.6 & 86.5 & $79.5^{\#}$ & $85.4^{*}$ & $17.9^{\circ}$ & $25.3^{\dagger \oplus}$ & $4.7^{\#}$ & 5.9 \\
\hline \multicolumn{9}{|l|}{ Hospital type } \\
\hline Type I & 78.0 & $85.4^{\ddagger}$ & 84.6 & $84.9^{\ddagger}$ & 21.1 & 24.5 & $4.7^{\S}$ & $5.9^{\S}$ \\
\hline Type II & 73.9 & $89.4^{\ddagger \S}$ & $77.1^{\circ}$ & $87.2^{*}$ & 21.1 & $37.0^{\ddagger \#}$ & 7.3 & 10.1 \\
\hline
\end{tabular}

Abbreviation: eGFR, estimated glomerular filtration rate.

${ }^{*} P<0.05,{ }^{\dagger} P<0.01, \stackrel{\ddagger}{\stackrel{\ddagger}{P}}<0.001$, compared with that of the first survey; ${ }^{\circledR} P<0.05$, ${ }^{\#} P<0.01,{ }^{\circledR} P<0.001$, compared with other groups of the same survey.

\section{DISCUSSION}

$\mathrm{CKD}$ is becoming a worldwide public health problem not only in developed countries but also in developing countries. ${ }^{23-25}$ Although much evidence has shown that BP control attenuates the rate of GFR decline and reduces the rate of cardiovascular complications in CKD patients, only limited data have been reported about hypertension awareness, treatment and control in this special population. Furthermore, most of this information has come from developed countries. $^{18-20}$ The present study provides data for hypertension awareness, treatment and control in CKD patients with hypertension in China over a 5-year period. To our knowledge, this is the first consecutive report from the developing world.
Our data demonstrate that hypertension awareness, treatment and control among hypertensive CKD patients in China increased over a 5 -year period. Recent data from a subgroup analysis of the Kidney Early Evaluation Program (KEEP) study, which was conducted among 10813 CKD patients, ${ }^{18}$ showed that $86.2 \%$ of them were identified as hypertensive at the $130 / 80 \mathrm{~mm} \mathrm{Hg}$ threshold, and only 34 and $13.2 \%$ of them had BP less than $140 / 90$ and $130 / 80 \mathrm{~mm} \mathrm{Hg}$, respectively. Similar results were reported in an Italian study, ${ }^{19}$ with 40.6 and $12.5 \%$ control for less than $140 / 90$ and $130 / 80 \mathrm{~mm} \mathrm{Hg}$, respectively. Data from NHANES $1988-1994^{20}$ showed that among patients with elevated Scr (men: Scr $\geqslant 1.6 \mathrm{mg}$ per $100 \mathrm{ml}$, women: $\mathrm{Scr} \geqslant 1.4 \mathrm{mg}$ per $100 \mathrm{ml}$ ) and hypertension, only $27 \%$ had BP less than $140 / 90 \mathrm{~mm} \mathrm{Hg}$. 
In our study, the control rate in the 2004-2005 survey for less than $140 / 90 \mathrm{~mm} \mathrm{Hg}$ was $34.8 \%$, which was significantly improved from that of the 1999-2000 survey and similar to that of the KEEP study. Admittedly, our participants were all enrolled in hospitals, which could contribute to the relatively high treatment and control rates, while participants in the KEEP study were enrolled by screening people at high risk for CKD. In addition, optimal hypertension control at the less than $130 / 80 \mathrm{~mm} \mathrm{Hg}$ threshold in both of our surveys was still poor and lower than that reported for developed countries, which is still not optimal.

Perhaps the most inspiring finding from the comparison of these two surveys is the improvement in BP control achieved by general physicians in local hospitals. Further analysis excluded bias due to clinical characteristics, and the improvement seemed, at least in part, to come from improved awareness and greater adoption of combined therapy. This indicates that more and more general physicians realized the importance of hypertension control for CKD patients and initiated education and treatment of their patients. Additionally, they tried to optimize therapy by using combined treatment for even greater control. Randomized clinical trials of patients with CKD have shown that adequate BP control is possible, but it requires close follow-up and, on average, three or four antihypertensive medications. ${ }^{26-28}$ Thus, improvement in hypertension management by general physicians is quite meaningful, given that most CKD patients are followed by general physicians rather than nephrologists in the current Chinese medical system.

In addition to clarifying the factors contributing to overall improvements, the surveys also raised some concerns that should be addressed. Awareness, in part, is a prerequisite for patient compliance with longterm treatment and may ultimately determine treatment efficacy to some degree. Still, there were approximately $13 \%$ of patients who were unaware of their hypertension in the 2004-2005 survey. Furthermore, young participants ( $<45$ years), who have the longest life expectancy, had the lowest awareness rates in both surveys, which might result in less treatment and less optimal BP control in this group. Patients' recognition of the importance of BP control also influences their compliance with long-term treatment and treatment efficacy. Notably, approximately $20 \%$ of patients in both surveys were untreated, with the leading reason being an unrecognized need for hypertensive treatment by the patient. This highlights the importance of hypertension education for CKD patients in daily clinical practice. In addition, the poor control rates for optimal BP control (less than $130 / 80 \mathrm{~mm} \mathrm{Hg}$ ) indicate, in part, insufficient acknowledgement by physicians of the more strict BP control needed for CKD patients. This stricter requirement must be emphasized in the future education of physicians.

This study was limited in that the surveys were cross-sectional and were conducted in relatively prosperous cities in China with large to moderate populations, such that only a few patients were recruited from rural and impoverished areas. Thus, the true overall awareness, treatment and control rates of hypertensive CKD patients in China may be lower than those reflected in the current data.

In conclusion, this is the first consecutive report of hypertension awareness, treatment and control of hypertensive CKD patients from a developing country. Improvement in hypertension awareness and BP control at a threshold less than $140 / 90 \mathrm{~mm} \mathrm{Hg}$ has been achieved in China, primarily because of the efforts of general physicians in local hospitals. Future strategies should focus on educating both physicians and CKD patients to achieve better adherence to optimal BP control at less than $130 / 80 \mathrm{~mm} \mathrm{Hg}$ and helping clinicians choose appropriate combination regimens to achieve strict $\mathrm{BP}$ control.

\section{CONFLICT OF INTEREST}

The authors declare no conflict of interest.

\section{ACKNOWLEDGEMENTS}

The surveys were supported by grants-in-aid for scientific research (985 projects) from the Ministry of Education Foundation of China.

1 Shulman NB, Ford CE, Hall WD, Blaufox MD, Simon D, Langford HG, Schneider KA. Prognostic value of serum creatinine and effect of treatment of hypertension on renal function. Results from the hypertension detection and follow-up program. The Hypertension Detection and Follow-Up Program Cooperative Group. Hypertension 1989; 13(Suppl 5): 180-193.

2 Walker WG, Neaton JD, Cutler JA, Neuwirth R, Cohen JD. Renal function change in hypertensive members of the Multiple Risk factor Intervention Trial. Racial and treatment effects. The MRFIT Research Group. J Am Med Assoc 1992; 268: 3085-3091.

3 Ruggenenti P, Perna A, Gherardi G, Benini R, Remuzzi G. Chronic proteinuric nephropathies: outcomes and response to treatment in a prospective cohort of 352 patients with different patterns of renal injury. Am J Kidney Dis 2000; 35: 1155-1165.

4 Garg AX, Clark WF, Haynes RB, House AA. Moderate renal insufficiency and the risk of cardiovascular mortality: results from the NHANES I. Kidney Int 2002; 61: 1486-1494.

5 Cinotti GA, Comunian C. Hypertension and renal disease. Ren Fail 1993; 15: 373-377.

6 Lewis EJ, Hunsicker LG, Bain RP, Rohde RD. The effect of angiotensin-convertingenzyme inhibition on diabetic nephropathy. The Collaborative Study Group. N Engl J Med 1993; 329: 1456-1462.

7 Lewis JB, Berl T, Bain RP, Rohde RD, Lewis EJ. Effect of intensive blood pressure control on the course of type 1 diabetic nephropathy. Collaborative Study Group. Am J Kidney Dis 1999; 34: 809-817.

8 Parving HH, Rossing P. The use of antihypertensive agents in prevention and treatment of diabetic nephropathy. Curr Opin Nephrol Hypertens 1994; 3: 292-300.

9 Parving $\mathrm{HH}$. Benefits and cost of antihypertensive treatment in incipient and overt diabetic nephropathy. J Hypertens Supp/ 1998; 16: S99-S101.

10 Ruggenenti P, Perna A, Gherardi G, Gaspari F, Benini R, Remuzzi G. Renal function and requirement for dialysis in chronic nephropathy patients on long-term ramipril: REIN follow-up trial. Lancet 1998; 352: 1252-1256.

11 Peterson JC, Adler S, Burkart JM, Greene T, Hebert LA, Hunsicker LG, King AJ, Klahr S, Massry SG, Seifter JL. Blood pressure control, proteinuria, and the progression of renal disease. The Modification of Diet in Renal Disease Study. Ann Intern Med 1995; 123: 754-762.

12 Brenner BM, Cooper ME, de Zeeuw D, Keane WF, Mitch WE, Parving HH, Remuzzi G, Snapinn SM, Zhang Z, Shahinfar S, RENAAL Study Investigators. Effects of losartan on renal and cardiovascular outcomes in patients with type 2 diabetes and nephropathy. $N$ Engl J Med 2001; 345: 861-869.

13 Curtis BM, Levin A, Parfrey PS. Multiple risk factor intervention in chronic kidney disease: control of cardiac disease in chronic kidney disease patients. Med Clin North Am 2005; 89: 511-523.

14 Ruilope LM, Salvetti A, Jamerson K, Hansson L, Warnold I, Wedel H, Zanchetti A. Renal function and intensive lowering of blood pressure in hypertensive participants of the hypertension optimal treatment (HOT) study. J Am Soc Nephrol 2001; 12: 218-225.

15 Hansson L, Zanchetti A, Carruthers SG, Dahlof B, Elmfeldt D, Julius S, Ménard J, Rahn $\mathrm{KH}$, Wedel $\mathrm{H}$, Westerling S. Effects of intensive blood pressure lowering and low dose aspirin in patients with hypertension: principal results of the Hypertension Optimal Treatment (HOT) randomised trial. Lancet 1998; 351: 1755-1762.

16 Chobanian AV, Bakris GL, Black HR, Cushman WC, Green LA, Izzo Jr JL, Jones DW, Materson BJ, Oparil S, Wright Jr JT, Roccella EJ, Joint National Committee on Prevention, Detection, Evaluation, and Treatment of High Blood Pressure. National Heart, Lung, and Blood Institute; National High Blood Pressure Education Program Coordinating Committee. Seventh report of the Joint National Committee on Prevention, Detection, Evaluation, and Treatment of High Blood Pressure. Hypertension 2003; 42: $1206-1252$.

17 Kidney Disease Outcomes Quality Initiative (K/DOQI). K/DOQI clinical practice guidelines on hypertension and antihypertensive agents in chronic kidney disease. $\mathrm{Am} \mathrm{J}$ Kidney Dis 2004; 43(Suppl 1): S1-S290.

18 Sarafidis PA, Li S, Chen SC, Collins AJ, Brown WW, Klag MJ, Bakris GL. Hypertension awareness, treatment, and control in chronic kidney disease. Am J Med 2008; 121 : 332-340.

19 De Nicola L, Minutolo R, Gallo C, Zoccali C, Cianciaruso B, Conte M, Lupo A, Fuiano G, Gallucci M, Bonomini M, Chiodini P, Signoriello G, Bellizzi V, Mallamaci F, Nappi F, Conte G. Management of hypertension in chronic kidney disease: the Italian multicentric study. J Nephrol 2005; 18: 397-404.

20 Coresh J, Wei GL, McQuillan G, Brancati FL, Levey AS, Jones C, Klaq MJ. Prevalence of high blood pressure and elevated serum creatinine level in the United States: Findings from the third National Health and Nutrition Examination Survey (1988-1994). Arch Intern Med 2001; 161: 1207-1216. 
21 Kidney Disease Outcome Quality Initiative. K/DOQI clinical practice guidelines for chronic kidney disease: evaluation classification stratification. Am J Kidney Dis 2002; 39(Suppl 2): S1-S246.

22 Ma YC, Zuo L, Chen JH, Luo Q, Yu XQ, Li Y, Xu JS, Huang SM, Wang LN, Huang W, Wang M, Xu GB, Wang HY. Modified glomerular filtration rate estimating equation for Chinese patients with chronic kidney disease. J Am Soc Nephrol 2006; 17: 2937-2944.

23 Coresh J, Byrd-Holt D, Astor BC, Briggs JP, Eggers PW, Lacher DA, Hostetter TH. Chronic kidney disease awareness, prevalence, and trends among US adults, 1999-2000. J Am Soc Nephrol 2005; 16: 180-188.

24 Zhang LX, Zuo L, Xu GB, Wang F, Wang M, Wang S, Lv J, Liu L, Wang H. Community-based screening for chronic kidney disease among population older than 40 years in Beijing. Nephrol Dial Transplant 2007; 22: 10931099.
25 Barsoum RS. Chronic kidney disease in the developing world. N Engl J Med 2006; 354: 997-999.

26 Peterson JC, Adler S, Burkart JM, Greene T, Hebert LA, Hunsicker LG, King AJ, Klahr S, Massry SG, Seifter JL. Blood pressure control, proteinuria, and the progression of renal disease. The Modification of Diet in Renal Disease Study. Ann Intern Med 1995; 123: 754-762.

27 Wright Jr JT, Bakris G, Greene T, Agodoa LY, Appel LJ, Charleston J, Cheek D, DouglasBaltimore JG, Gassman J, Glassock R, Hebert L, Jamerson K, Lewis J, Phillips RA, Toto RD, Middleton JP, Rostand SG. Effect of blood pressure lowering and antihypertensive drug class on progression of hypertensive kidney disease: results from the AASK trial. J Am Med Assoc 2002; 288: 2421-2431.

28 Parikh NI, Hwang SJ, Larson MG, Meigs JB, Levy D, Fox CS. Cardiovascular disease risk factors in chronic kidney disease: overall burden and rates of treatment and control. Arch Intern Med 2006; 166: 1884-1891. 